\title{
Fast flow phenomena in a toroidal plasma*
}

\author{
D. J. Den Hartog, ${ }^{\dagger}$ A. F. Almagri, J. T. Chapman, H. Ji, S. C. Prager, and J. S. Sarff \\ Department of Physics, University of Wisconsin-Madison, 1150 University Avenue, Madison, \\ Wisconsin 53706 \\ R. J. Fonck and C. C. Hegna \\ Department of Nuclear Engineering and Engineering Physics; University of Wisconsin-Madison, \\ 1500 Johnson Drive, Madison, Wisconsin 53706
}

(Received 14 November 1994; accepted 30 January 1995)

The bulk fluid velocity is measured spectroscopically with $10 \mu$ s time resolution in the Madison Symmetric Torus (MST) reversed-field pinch (RFP) [Fusion Technol. 19, 131 (1991)], a diagnostic capability used to study the fast flow dynamics associated with locked modes and the RFP dynamo. The phase velocity of the tearing modes and the fluid velocity accelerate between sawtooth events, reaching a maximum speed of about $20 \mathrm{~km} / \mathrm{s}$ in a few ms. Both slow down at the sawtooth crash in $\approx 100 \mu$ s. This deceleration time scale is as calculated for the tearing modes from the action of electromagnetic torque on the magnetic islands, but is much faster than expected from the viscous torque on the bulk fluid. In the RFP, correlated fluctuations in the tearing modes and fluid velocity probably also generate current via the "RFP dynamo," $\langle\tilde{\mathbf{u}} \times \tilde{\mathbf{B}}\rangle$, where $\mathbf{u}$ is the bulk fluid velocity. Initial data indicate a possible increase in $\left\langle\tilde{u}_{\phi} \tilde{B}_{r}\right\rangle$ during sawtooth events, coincident with toroidal flux generation. (ㅇ) 1995 American Institute of Physics.

\section{INTRODUCTION}

The flow velocity dynamics of ions in magnetically confined plasmas figure prominently in a variety of plasma phenomena, including particle transport, ${ }^{1}$ high $(\mathrm{H})$-mode operation, ${ }^{2}$ locked modes, ${ }^{3-5}$ and the reversed-field pinch (RFP) dynamo. ${ }^{6,7}$ In this paper, we focus on the dynamics and interdependence of plasma flow, magnetic fluctuations, and magnetic mode rotation. Particular attention will be paid to the fast flow dynamics during "wall locking," in which the helical magnetic structure in the plasma suddenly ceases rotation in response to a perturbation of the confirming magnetic field (a "field error"). We report the first fast time scale $(10 \mu \mathrm{s})$ measurements of the directed flow velocity of impurity ions in a toroidal high-temperature plasma. These measurements illustrate the close coupling between the toroidal rotation of the magnetic modes and the plasma flow. Both the modes and the plasma decelerate in about $100 \mu$ s during a locking event. This deceleration of the modes is as expected from a calculation of the electromagnetic torque on the magnetic islands, but a simple calculation of the viscous torque on the plasma predicts a much longer deceleration time. The acceleration of modes and plasma following unlocking occurs on the slower millisecond particle confinement time scale. High-speed measurements of flow velocity fluctuations, when correlated with the magnetic field fluctuations, also indicate that the RFP magnetic field configuration may be sustained against resistive diffusion by the RFP dynamo.

In this paper we begin with a short description of the experimental apparatus. The main body of the paper is a description of the experimental phenomenology of flow and locking, which is discussed in the dynamical context of the torque balance on an island. Our initial study of the RFP

\footnotetext{
${ }^{*}$ Paper 8IB1, Bull. Am. Phys. Soc. 39, 1721 (1994).

Invited speaker.
}

dynamo is described in a short section prior to the summary.

\section{APPARATUS}

These studies were performed on the Madison Symmetric Torus $(\mathrm{MST})^{8,9}$ reversed-field pinch $(\mathrm{RFP})^{10}$ toroidal plasma confinement device. MST has a minor radius $a=0.52$ $\mathrm{m}$ and a major radius $R=1.5 \mathrm{~m}$. Typical parameters are a plasma current of $350 \mathrm{kA}$, central electron temperature of $250 \mathrm{eV}$, central ion temperature of $180 \mathrm{eV}$, line-averaged electron density of $1.5 \times 10^{19} \mathrm{~m}^{-3}$, and a discharge duration of $60 \mathrm{~ms}$. Hydrogen is the majority species in the plasma, with carbon and oxygen (the two major impurities) contamination at the level of $1 \%-2 \%$.

We have developed a specialized spectroscopic diagnostic system for the measurement of plasma ion dynamics. This system combines good resolution and throughput with highspeed parallel light collection. Measurement precision of $<0.6 \mathrm{~km} / \mathrm{s}$ has been demonstrated, with a digitization rate of $100 \mathrm{kHz}$ and a corresponding analog bandwidth of $35 \mathrm{kHz}$. Only a brief description of the hardware is given here, as this system and its calibration have been described in detail elsewhere. ${ }^{11,12}$ The measurement technique is based on highspeed passive Doppler spectroscopy of $\mathrm{CV}$ impurity ions $(227.091 \mathrm{~nm})$ that are localized near the hot core of the MST plasma. Our instrument simultaneously records two opposing toroidal chordal views of the plasma, for measurement of toroidal flow velocity $\tilde{u}_{\phi}$. Each view is dispersed over 16 spectral wavelength channels. The chordal views inherent to passive spectroscopy limit the spatial resolution, but this is acceptable on MST because the magnetohydrodynamic (MHD) phenomena of interest are large scale, with wavelengths on the order of $2 \pi R / n$ (which for MST with the dominant toroidal mode number $n=6$ is about equal to $R$ ).

We believe that the $\mathrm{C} v$ impurity ions in MST reflect the behavior of the majority species (and thus the magnetohydrodynamic behavior of the plasma) for several reasons. 
First, the temperatures of the $\mathrm{CV}$ ions and the majority protons (measured with a charge-exchange analyzer) are similar, both in magnitude and fast time behavior. ${ }^{13}$ Second, for typical MST parameters, the calculated energy equilibration time for the carbon impurity ions with the majority protons is approximately $50 \mu \mathrm{s.} .{ }^{14}$ Finally, the theoretical prediction of $\mathrm{Kim}^{15}$ that the flow of impurity and majority ions will be different does not apply to MST because neoclassical effects are small in a RFP plasma.

MST has an extensive set of magnetic diagnostics in the form of arrays of flux pickup coils monnted on the inner wall of the vacuum vessel. There are large poloidally and toroidally distributed sets of three orthogonal coils for measuring tearing mode structure and rotation. The radial field error at the poloidal and toroidal insulated $1 \mathrm{~cm}$ wide gaps in the conducting shell surrounding the plasma are also monitored by arrays of coils. (The poloidal gap extends the short way around the torus to allow the pulsed poloidal field to enter the conducting shell; the toroidal gap extends the long way around the torus to allow application of the toroidal field. ${ }^{16}$ ) In addition, the $\tilde{B}_{r}$ measurements necessary for study of the RFP dynamo were made with a probe that contained an array of flux coils and was shielded with boron nitride so that it could be inserted into low current (250 kA) MST discharges.

\section{FLOW AND LOCKING}

Sections III A and III D below are a presentation of the experimental phenomenology of flow and locking in MST; Secs. III B and III C are a review of the theoretical context in which we have placed our work.

\section{A. The locking sequence}

The dominant magnetic tearing modes in MST (with poloidal and toroidal mode numbers $m=1, n=5-10$ ) phase lock to form a rotating, localized, magnetic disturbance with a toroidal width of about one-fourth the circumference. ${ }^{16}$ The condition we refer to as "wall-locked modes" occurs when this rotating magnetic structure halts at the physical location of the radial error field; this always occurs coincident with a sawtooth event. (MST exhibits distinct periodic sawtooth events that are evident in many plasma parameters, including soft $\mathrm{x}$-ray emission, plasma current, toroidal fiux, and majority ion temperature. ${ }^{13,17}$ ) The sequence of events during wall locking is as follows.

(1) The sudden flattening of the current density profile that occurs during a sawtooth event shifts the plasma inward and generates an impulsive radial magnetic field error at the poloidal gap.

(2) The rotating, phase-locked tearing modes halt at the physical location of this error field.

(3) At this point the sequence bifurcates and is completed by either (a) temporary locking, which is characterized by slow reacceleration of the modes and plasma to $\approx 20$ $\mathrm{km} / \mathrm{s}$ and a gradual decrease in the field error; or (b) pernanent locking, in which the modes do not reaccelerate, the field error grows linearly, and the plasma reaccelerates or continues at $\approx 10 \mathrm{~km} / \mathrm{s}$ (which is approximately the velocity

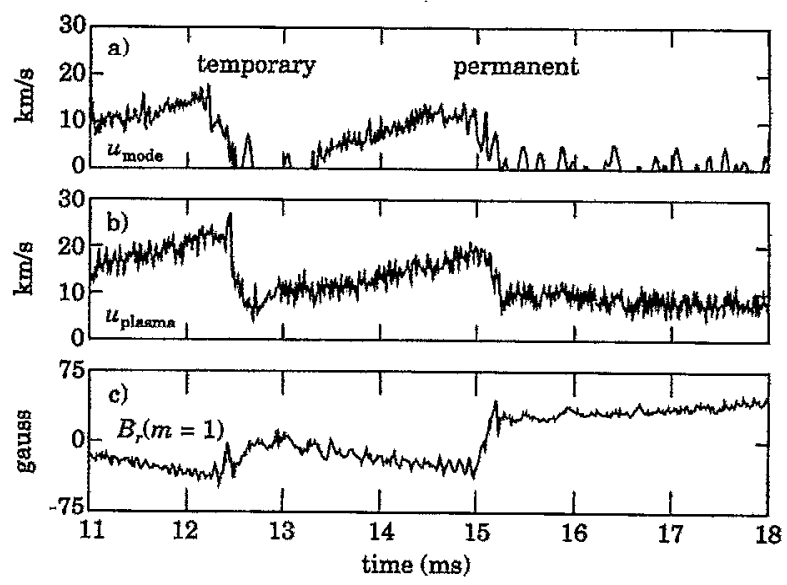

FIG. 1. Temporary locking followed by permanent locking, illustrated by two successive sawtooth events in MST. (a) The rotation of the $m=1, n=6$ tearing mode halts during the sawtooth, but reaccelerates after temporary locking. (b) The toroidal fiow velocity of the plasma slows down but often does not go to zero during locking. (c) The $m=1$ radial field error at the poloidal gap impulsively rises during a sawtooth event, but continues to linearly grow only after permanent locking.

expected from the $\mathbf{E} \times \mathbf{B}$ and ion diamagnetic drifts in $\mathrm{MST}^{18}$ ).

Both of these locking phenomena occurred in the single RFP discharge shown in Fig. 1. Temporary locking is by far the predominant mechanism. The cause of the bifurcation in the locking sequence is not known; it may be due to the type of field error instability described in Ref. 16.

\section{B. Torque balance on an island}

The framework in which we discuss our measurements of the plasma and mode rotation during wall locking is based on the supposition that such behavior is determined by the response of the magnetic islands in the MST plasma to the

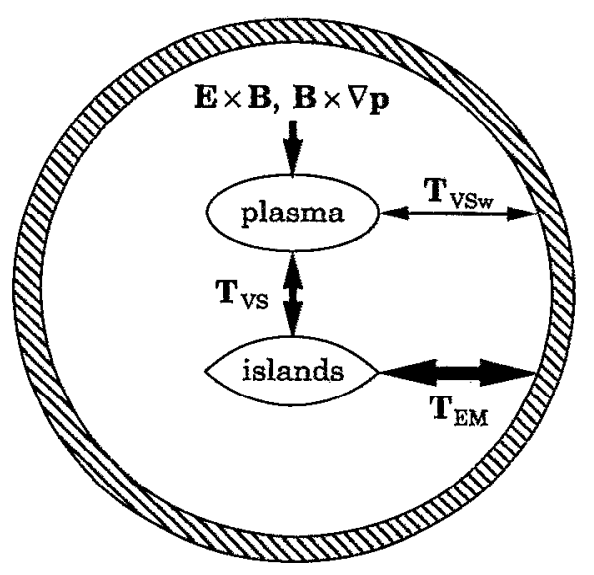

FIG. 2. A pictorial overview of the forces on the plasma and islands. The $\mathbf{E} \times \mathbf{B}$ and $\mathbf{B} \times \nabla \mathbf{p}$ forces accelerate the bulk plasma; the plasma is decelerated by the viscous drag against the wall, $\mathbf{T}_{V S_{w}}$. The islands are decelerated by the electromagnetic torque imposed by the field error, $\mathbf{T}_{\mathrm{EM}}$. The plasma and islands interact to decelerate and aceelerate each other via viscous coupling, $\mathbf{T}_{\mathrm{VS}}$. 
perturbing field error. Specifically, the inertia of the plasma inside the separatrices of the magnetic islands must be balanced against the local electromagnetic and viscous torques. ${ }^{19,20}$ Figure 2 is a pictorial overview of this process, which may be helpful in understanding the discussion below.

The electromagnetic force on the plasma in a rotating island is

$$
\mathbf{F}_{\mathrm{EM}} \approx \tilde{\mathbf{J}} \times \tilde{\mathbf{B}} \text {, }
$$

where $\tilde{\mathbf{J}} \approx \tilde{\mathbf{J}}_{\mathrm{fe}}$, the current at the resonant surface generated by the impulsive field error, and $\tilde{\mathbf{B}}$ is the preexisting fluctuating magnetic field. Then, the torque on an island is

$$
\mathbf{T}_{\mathrm{EM}}=\int r(\tilde{\mathbf{J}} \times \tilde{\mathbf{B}}) d S \approx \int r\left(\tilde{\mathbf{J}}_{\mathrm{fe}} \times \tilde{\mathbf{B}}\right) d S,
$$

where $r$ can be the major or minor radius for toroidal or poloidal torques and the integral is over the surface of the island.

In order to estimate the viscous torque $\mathbf{T}_{\mathrm{Vs}}$ on the islands, they can be treated as solid obstacles in an incompressible fluid. In the inner region of the rational surfaces,

$$
\mathbf{T}_{\mathrm{VSs}} \propto \mu_{\perp} \frac{\partial \Omega_{s}}{\partial r}
$$

where $\Omega_{s}$ is the angular velocity at the rational surface and $\mu_{\perp}$ is the perpendicular viscosity. The angular velocity is

$$
\Omega_{s}=\frac{\mathbf{k} \cdot \mathbf{v}}{r}=\frac{n u_{\phi}}{R}+\frac{m u_{\theta}}{a} .
$$

An analysis of this dispersion equation for magnetic mode rotation in MST suggests that $u_{\phi} \geqslant u_{\theta}$; therefore $\Omega_{s} \approx n u_{\phi} / R$ because $n>m$. (The poloidal flow velocity $u_{\theta}$ will be measured on MST in the near future when the appropriate viewing port has been constructed.) The perpendicular viscosity is $^{21,22}$

$$
\mu_{\perp}=\frac{6 n k T_{i}}{\omega_{c i}^{2} \tau_{i}},
$$

where $n$ is the ion density, $k$ is Boltzmann's constant, $T_{i}$ is the ion temperature, $\omega_{c i}$ is the ion cyclotron frequency, and $\tau_{i}$ is the ion collision time.

The equation of motion of the island is then obtained by balancing inertia against the torques:

$$
\rho\left(\frac{\partial \Omega_{s}}{\partial t}\right) \propto \mathbf{T}_{\mathrm{VSs}}+\mathbf{T}_{\mathrm{EMs}} .
$$

\section{Threshold for locking and unlocking}

The size of the perturbing field error necessary to cause wall locking can be calculated by noting that the locked state is stationary and requires $\mathbf{T}_{\mathrm{VSs}}=\mathbf{T}_{\mathrm{EM} s}$. Estimating the electromagnetic torque,

$$
\begin{aligned}
\mathbf{T}_{\mathrm{EM} \dot{s}} & \propto\left\langle\tilde{\mathbf{J}}_{\mathrm{fe}} \times \tilde{\mathbf{B}}\right\rangle \\
& \propto \tilde{B}_{v} \tilde{B} \sin \Delta \varphi \\
& \propto W_{v}^{2} W^{2} \sin \Delta \varphi
\end{aligned}
$$

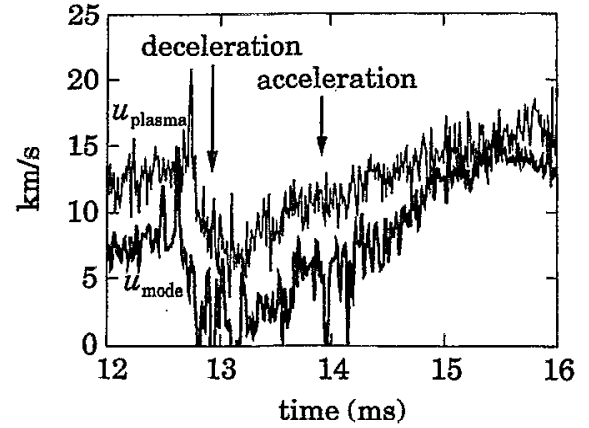

FIG. 3. A temporary locking cycle over one sawtooth period; the starting points of the deceleration and acceleration phases are illustrated. Both the plasma (light line) and mode (heavy line) toroidal velocities are shown.

where $\Delta \varphi$ is the phase between $\tilde{\mathbf{J}}_{\mathrm{fe}}$ and $\tilde{\mathbf{B}}, W$ indicates the island width, and the subscript " $v$ " indicates a vacuum quantity (e.g., $B_{v}$ is the field error that exists in the vacuum vessel prior to formation of the plasma). At the locking threshold, $\sin \Delta \varphi=1$, so balancing the torques gives

$$
\mu_{\perp} \frac{\Omega}{W} \propto W_{v}^{2} W^{2},
$$

therefore,

$$
W_{v} \propto \sqrt{\mu_{\perp} \frac{\Omega}{W^{3}}} .
$$

For locking to occur in MST, $W_{v} \approx 0.5 \mathrm{~cm}$ or a vacuum error field of $\approx 2 \%$ at the poloidal gap, about what is observed. (The vacuum field error is actually a spatial $\delta$ function localized at the $1 \mathrm{~cm}$ wide poloidal gap.)

At the unlocking threshold, the flow velocity, profile has relaxed, so

$$
\mu_{\perp} \frac{\Omega}{r_{s}} \propto W_{v}^{2} W^{2}
$$

and

$$
\frac{W_{\text {vulock }}}{W_{\text {vlock }}} \propto \frac{W}{r_{s}}<1,
$$

where $r_{s}$ is the radius of the rational surface.

\section{The temporary locking cycle}

Figure 3 illustrates a typical temporary locking cycle in MST; it is an expanded view of the plasma and mode toroidal rotation velocity during one sawtooth period.

\section{The deceleration phase}

Deceleration of both the magnetic modes and plasma during wall locking is quick, on the order of $100 \mu$ s. Since the island and thus the mode deceleration is caused by the electromagnetic braking torque imposed by the field error, it is straightforward to show that the expected mode deceleration time scale is 


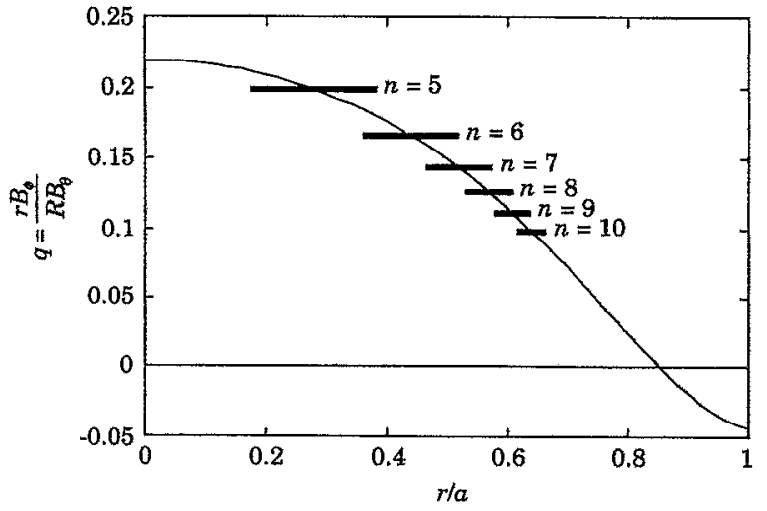

FIG. 4. Island widths in MST as estimated from $\tilde{B}$ measured at $r=a$.

$$
\begin{aligned}
\tau_{M} & =\tau_{H} \sqrt{\frac{r_{s} R_{0}^{2}}{W W_{v}^{2}}} \\
& \approx 300 \mu \mathrm{s} .
\end{aligned}
$$

The Alfvén time is

$$
\tau_{H}=\frac{l_{s}}{m v_{\mathrm{A}}}
$$

where $v_{\mathrm{A}}$ is the Alfvern velocity, $m$ is the poloidal mode number, and the shear length ( $q$ is the safety factor) is

$$
l_{s}=\frac{R q^{2}}{a(d q / d r)} .
$$

The value of $\tau_{M}$ shown is for typical MST parameters of $\tau_{H} \approx 1 \mu \mathrm{s}, r_{s} \approx 25 \mathrm{~cm}, W \approx 10 \mathrm{~cm}$, and $W_{v} \approx 0.5 \mathrm{~cm}$. Encouraged by this agreement with experiment, we calculate the viscous flow deceleration of the bulk plasma:

$$
\begin{aligned}
\tau_{\mathrm{VS}} & \approx \frac{a^{2}}{\nu(2.4)^{2}} \\
& \approx \frac{1}{6} \tau_{v}\left(\frac{a}{r_{s}}\right)^{2} \\
& \approx 0.13 \mathrm{~s},
\end{aligned}
$$

where $\tau_{v}=r_{s}^{2} / \nu$ is the viscous time scale and $\nu=\mu_{\perp} / \rho$ ( $\rho$ is the mass density).

This is obviously much longer than the measured deceleration time of the plasma. The cause of this disagreement is unclear, but a possible explanation lies in the fact that the $m=1$ islands in MST are large and overlapping; see Fig. 4 for the estimated island widths and positions. Since most of the plasma cross section is covered by islands, we may expect to see the entire plasma decelerate on the electromagnetic braking time scale, even though it may actually be viscous torques that act on the bulk plasma.

\section{The acceleration phase}

Referring again to Fig. 3, note that reacceleration to the equilibrium speed of about $20 \mathrm{~km} / \mathrm{s}$ takes several milliseconds. This is roughly equivalent to the particle confinement time in MST. We suggest the following scenario.
(1) Large transport of energy and particles takes place during a sawtooth event (density and temperature profiles fiatten, potential gradients are lost).

(2) A radial electric field is reestablished by particle diffusion on a particle confinement time scale.

(3) The bulk plasma accelerates in response to the $\mathrm{E} \times \mathbf{B}$ force.

(4) Momentum transfer to the islands then accelerates the modes.

During a sawtooth event, the mode velocity always drops to zero while the plasma velocity often drops to about $5 \mathrm{~km} / \mathrm{s}$ and then starts to accelerate. It then takes several milliseconds for the modes to "catch up" to the plasma, that is, for the modes to accelerate to the same velocity as the plasma. When the two velocities again match, we sometimes see a 50\% drop in the amplitude of the $m=1, n=6$ tearing mode (which is the largest mode during that phase of the sawtooth). There is no accompanying rise in the amplitude of the other tearing modes. The cause of this behavior is under investigation.

\section{INITIAL STUDY OF THE RFP DYNAMO}

Extensive MHD computation has shown that current is driven by correlated fluctuations in the plasma flow velocity and the magnetic field (this is the RFP dynamo). ${ }^{23}$ This mechanism is believed to sustain the reversed toroidal field profile of the RFP plasma against resistive diffusion. The dynamo is compatible with relaxation through resistive reconnection to the minimum energy "Taylor state." 24 The RFP dynamo can be viewed as a fluctuation-induced electric field, $\mathbf{E}_{f}=(\tilde{\mathbf{u}} \times \tilde{\mathbf{B}}) ; \mathbf{E}_{f}$ drives parallel current $\left(J_{\theta}\right)$ in the outer plasma and impedes parallel current $\left(J_{\phi}\right)$ in the center. The dynamo current drive in the outer region of the plasma causes $B_{\phi}$ there to reverse direction relative to $B_{\phi}$ in the center.

Probe studies of the edge plasma in MST have inferred the existence of the RFP dynamo from measurement of the

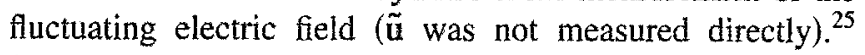
Spectroscopic measurement of $\mathrm{OV}$ velocity fuctuations in the Reversed-field Pinch, University of Tokyo Experiment (REPUTE)- $1^{26}$ RFP also points to the existence of the $d y$ namo, although the correlation between $\tilde{u}$ and $\tilde{B}$ was not measured. ${ }^{27}$ Our measurements in the core plasma also show that large fluctuations in $\tilde{u}_{\phi}$ and $\tilde{B}_{r}$ occur during a sawtooth event, coincident with the generation of $B_{\phi}$ (thus making a sawtooth event a "discrete dynamo event" ${ }^{\prime 17}$ ). Part of these fluctuations are due to a rigid shift of the plasma column during a sawtooth, but we use a data reduction algorithm (Ref. 12), which quantifies the actual flux-surface average of the random fluctuations in $\tilde{u}_{\phi}$ and $\tilde{B}_{r}$ from an ensemble of sawtooth events. Figure 5 shows the correlation of $\tilde{u}_{\phi}$ with $\tilde{B}_{r}$ measured at $r / a=0.6\left(\tilde{u}_{\phi}\right.$ is measured from the $\mathrm{C}$ V shell between $r / a=0.3$ and 0.6 ) from our small initial ensemble containing about 100 sawtooth events. These measurements show what may be an increase in $\mathbf{E}_{f}=\left\langle\tilde{u}_{\phi} \tilde{B}_{r}\right\rangle$ during the sawtooth event. 

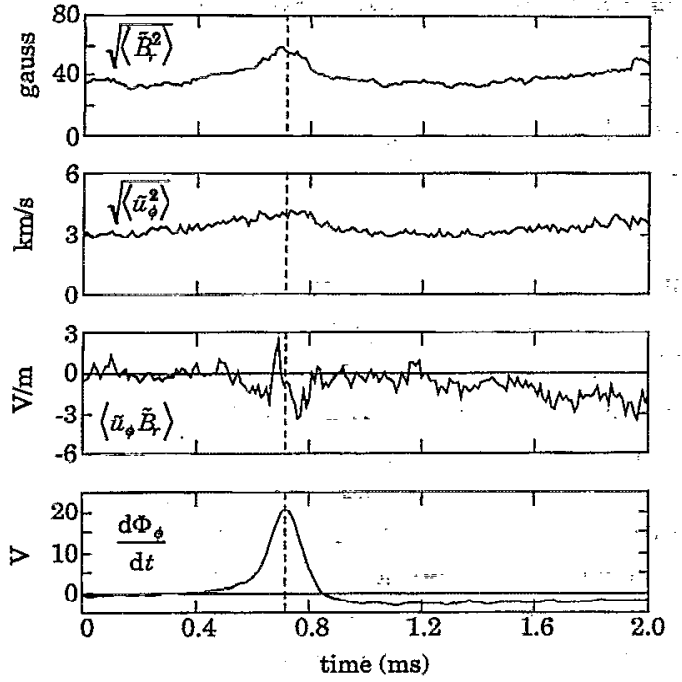

FIG. 5. The RMS values of $\tilde{B}_{r}$ and $\tilde{u}_{\phi}$ and the correlation of $\tilde{u}_{\phi}$ with $\tilde{B}_{r}$, measured at $r / a=0.6\left(\tilde{u}_{\phi}\right.$ is measured from the $\mathrm{CV}$ shell hetween $r / a$ $=0.3$ and 0.6 ). The dashed line indicates the time at which toroidal flux generation during a sawtooth event is at a maximum; see the plot of $d \Phi_{\phi} / d t$.

\section{SUMMARY AND CONCLUSIONS}

Precise, high-speed passive measurements of the plasma flow velocity in MST show quick deceleration of both the rotating magnetic modes and the plasma during wall locking. The observed field error locking threshold and mode deceleration time scale are as expected from the action of electromagnetic torque on the magnetic islands in MST. However, the deceleration of the plasma following locking is much faster than expected from the viscous torque applied on the plasma by a wall-locked island structure. This disagreement may arise because the islands in MST are large and overlapping and may exert much more braking torque on the plasma than calculated from a simple application of the theory of viscous flow around an island. Acceleration of the plasma and modes following unlocking takes place on the slower particle confinement time scale, perhaps indicalive of the time needed to reestablish a radial electric field following a sawtooth/locking event.

Initial measurements of the $\left\langle\tilde{u}_{\phi} \tilde{B}_{r}\right\rangle$ term of the RFP dynamo in the core of MST indicate a possible increase during sawtooth events, coincident with toroidal flux generation.

Future work on MST will continue the study of the dynamo with a duospectrometer that has been upgraded with greater light throughput and now has a $250 \mathrm{kHz}$ fluctuation bandwidth. We plan to take large ensemble measurement of both the $\tilde{u}_{\phi} \tilde{B}_{r}$ and $\tilde{u}_{r} \tilde{B}_{\phi}$ dynamo terms. Studies of flow and locking will continue with active electrode biasing of the plasma to accelerate or brake the plasma:via a radial electric field. New light collection ports will also allow us to measure poloidal and radial (if it is primarily $m=1$ ) plasma flow.

\section{ACKNOWLEDGMENTS}

This work could not have been done without valuable contributions from the entire MST group, both the scientific and the technical staff.

This work is supported by the U. S. Department of Energy.

${ }^{\prime}$ H. Biglari, P, H. Diamond, and P. W. Terry, Phys. Fluids 31, 2644 (1988).

${ }^{2}$ R. J. Groebner, K. H. Burrell, and R. P. Seraydarian, Phys. Rev. Lett. 64, 3015 (1990).

${ }^{3}$ R. Fitzpatrick and T. C. Hender, Phys. Fluids B 3, 644 (1991).

${ }^{4}$ A. W. Morris, P. G. Carolan, R. Fitzpatrick, T. C. Hender, and T. N. Todd, Phys. Fluids B 4, 413 (1992).

${ }^{5}$ T. C. Hender, R. Fitzpatrick, A. W. Morris, P. G. Carolan, R. D. Durst, T. Edlington, J. Ferreira, S. J. Fielding, P. S. Haynes, J. Hugill, I. J. Jenkins, R. J. La Haye, B. J. Parham, D. C. Robinson, T. N. Tödd, M. Valovič, and G. Vayakis, Nucl. Fusion 32, 2091 (1992).

${ }^{6} \Lambda$. Sykes and J. A. Wesson, Proceedings of the 8th European Conference on Controlled Fusion and Plasma Physics, Prague, 1977 (European Physical Society, Petit-Lancy, 1977). Vol. I, p. 80.

${ }^{7}$ E. J. Caramana, R. A. Nebel, and D. D. Schnack, Phys. Fluids 26, 1035 (1983).

${ }^{8}$ R. N. Dexter, D. W. Kerst, T. W. Lovell, S. C. Prager, and J. C. Sprott, Fusion Technol. 19, 131 (1991).

${ }^{9} \mathrm{~S}$. Hokin, A. Almagri, M. Cekic, B. Chapman, N. Crocker, D. J. Den Hartog, G. Fiksel, J. Henry, H. Ji, S. Prager, J. Sarff, E. Scime, W. Shen, M. Stoneking, and C. Watts, J. Fusion Energy 12, 281 (1993).

${ }^{10}$ H. A. B. Bodin and A. A. Newton, Nucl. Fusion 19, 1255 (1980).

IID. J. Den Hartog and R. J. Fonck, Rev. Scí. Instrum. 65, 3238 (1994).

${ }^{12}$ D. J. Den Hartog, A. F. Almagri, R. J. Fonck, and S. C. Prager, Rev. Sci. Instrum. 66, 444 (1995)

${ }^{13}$ E. Scime, M. Cekic, D. J. Den Hartog, S. Hokin, D. J. Holly, and C. Watts, Phys. Fluids B 4, 4062 (1992).

${ }^{14}$ I. H. Hutchinson, Principles of Plasma Diagnostics (Cambridge University Press, Cambridge, 1987), p. 228.

${ }^{15}$ Y. B. Kim, P. H. Diamond, and R. J. Groebner, Phys. Fluids B 3, 2050 (1991).

${ }^{10}$ A. F. Almagri, S. Assadi, S. C. Prager, J. S. Sarff, and D. W. Kerst, Phys Fluids B 4, 4080 (1992).

${ }^{17}$ S. Hokin, A. Almagri, S. Assadi, J. Beckstead, G. Chartas, N. Crocker, M. Cudzinovic, D. Den Hartog, R. Dexter, D. Holly, R. Nebel, S. Prager, T. Rempel, J. Sarff, E. Scime, W. Shen, C. Spragins, C. Sprott, G. Starr, M. Stoneking, and C. Watts, Phys. Fluids B 3, 2241 (1991).

${ }^{18}$ M. R. Stoneking, S. A. Hokin, S. C. Prager, G. Fiksel, H. Ji, and D. J. Den Hartog, Phys. Rev. Lett. 73,549 (1994).

${ }^{19}$ R. Fitzpatrick, Nucl. Fusion 33, 1049 (1993).

${ }^{20}$ R. Fitzpatrick, R. J. Hastie, T. J. Martin, and C. M. Roach, Nucl. Fusion 33, 1533 (1993).

${ }^{21}$ J. D. Huba, Naval Research Laboratory Plasma Formulary (U. S. Department of the Navy, Naval Research Laboratory, Washington, DC, 1994), p. 38.

${ }^{22}$ S. I. Braginskii, Sov. Phys. JETP 6, 358 (1958).

${ }^{23}$ S. Ortolani and D. D. Schnack, Magnetohydrodynamics of Plasma Relaxation (World Scientific, Singapore, 1993), p. 95.

${ }^{24}$ J. B. Taylor, Phys. Rev. Lett. 33, 139 (1974); Rey. Mod. Phys. 58, 741 (1986)

${ }^{25}$ H. Ji, A. F. AImagri, S. C. Prager, and J. S. Sarff, Phys. Rev. Lett. 73, 668 (1994).

${ }^{26}$ N. Asakura, T. Fujita, K. Hattori, N. Inoue, S. Ishida, K. Kamada, S. Mattsuzuka, K. Miyamoto, J. Morikawa, Y. Nagayama, H. Nihei, S. Shinohara, H. Toyama, Y. Ueda, K. Yamagishi, and Z. Yoshida, Plasma Phys. Controlled Fusion 28, 805 (1986).

${ }^{27}$ A. Ejiri, S. Ohdachi, T. Oikawa, S: Shinohara, K. Yamagishi, H. Toyama, and K. Miyamoto, Phys. Plasmas 1, 1177 (1994) 